\title{
Effect of Soybean Leaf Protease Inhibitor on the Mean Leaf Area Consumed by Spodopetra litura and Spilosoma obliqua larvae
}

\author{
Anchala Nautiyal", Neeta Gaur and Kamendra Singh \\ Department of Entomology, College of Agriculture, G.B. Pant University of Agriculture and \\ Technology, Pantnagar-263145, U.S. Nagar, Uttarakhand, India \\ *Corresponding author
}

A B S T R A C T

\section{Keywords}

Spodopetra litura and Spilosoma obliqua larvae.

\section{Article Info}

Accepted:

12 September 2017

Available Online:

10 November 2017
The aim of the present investigation was to study the effect of soybean leaf protein and protease inhibitor content on the mean leaf area consumed by Spodoptera litura and Spilosoma obliqua larva. The activity of trypsin inhibitor was assayed by determining the residual trypsin activity by using BapNA as the substrate and bovine trypsin as the standard enzyme. The highest and lowest TIA activity exhibited by genotypes SL 979 and SL 688 (2.25 TUI.mg ${ }^{-1}$ protein) and CSB 904 (0.35 TUI. mg ${ }^{-1}$ protein), respectively. The highest gut trypsin inhibition activity ascertained in larvae fed on SL $688(14.40 \%)$ While the lowest intensity of gut trypsin inhibition activity was observed in genotypes NRC 94 $(0.12 \%)$. A highly significant and negative correlation was observed between MLAC $\left(\mathrm{cm}^{2}\right)$ by $S$. litura and $S$. obliqua and protein content in 33 , leaves $\left(\mathrm{r}=-0.728^{* *}\right)$ and $\left(-0.674^{* *}\right)$ respectively and trypsin inhibitor in leaves $\left(\mathrm{r}=-0.909^{* *}\right)$ and $\left(\mathrm{r}=-0.913^{* *}\right)$. Thus it can be concluded that the genotypes which were having higher protein and trypsin inhibitor in their leaves offered resistance against $S$. litura and $S$. boliqua in soybean.

\section{Introduction}

Plant protease inhibitors (PIs) are small proteins which bind with proteolytic enzymes and are widely spread throughout the plant kingdom, these are generally present in high concentration in storage tissues (up to $10 \%$ of protein content), and also detectable in leaves during several physiological processes, such as reserve control and defense against pathogens and insect pests (Koiwa et al., 1997). In the latter case, PIs have been shown to be developmentally expressed in seeds and reserve organs (Koiwa et al., 1997) or induced by wounding in leaves (Schaller and Ryan, 1995). The most common of these plant inhibitors are those inhibiting serine proteases such as trypsin (Ahmad et al., 1980; Sasaki and Suzuki, 1982; Hamad and Attias, 1987; Broadway, 1989; Houseman et al., 1989; Johnston et al., 1991). PIs efficiently inhibit elastase and trypsin-like activities from the larval midgut of Spodoptera littoralis leading to their starvation and subsequent death. Hence, mode of action and expression profile suggests that PIs is a factor of Soybean insect resistance. This fact can be used as a potential strategy for increasing the level of plant defnce against insects (Brik, 1995 and Koiwa et al., 1997). Many reports shows that different serine protease inhibitors have negative effect on the growth and 
development of lepidoptrous larvae (Shukle and Murdock). Therefore, it is important to biochemically characterized the protease inhibitors from various indigenous cultivated legumes and evaluate their insecticidal potential.

Soybean is a crop of global importance and is one of the most frequently cultivated crops worldwide. It suffers severe losses due to insect predation. Most of these losses caused by defoliators (Spodopetera litura). Gangrade (1976) reported over 99 insect species attacking soybean crop at Jabalpur. But now the situation has changed and as many as 275 insect species have been recorded attacking soybean crop in India. For this reason, it became important to assess the levels of protease inhibitors form soybean and there interaction with the gut protease of S. litura. In view of this the present work focus on the determination of the concentration of trypsin inhibitor in leaves of 33 genotypes of soybean, gut trypsin inhibition percent in $S$. litura and their correlation with the mean leaf area consumed by the larvae of S. litura and S. obliqua.

\section{Materials and Methods}

\section{Plant material}

Leaves of 33 verities viz. CSB-904, DS-2705, DS-2706, DS-2708, DSb-19, DSb-21, JS 2041, JS-20-69, JS 20-71, KBS 22-2009, KDS378, KDS-378, KDS-695, KDS-699, KDS705, KDS-708, MACS-1340, MACS-1394, MACS-1416, MAUS-612, MAUS-614,NRC92,NRC-93, NRC-94, PS-1518, PK-5113, RVS 2001-18, SL-958, SL-979, SL-982, SL688, PS-1092, PS-1347, SL-688 (SC), PS1092(SC), PS-1347(SC) of soybean were obtain from entomological block of Norman E. Borlaug Crop Research Centre of Govind Ballabh Pant University of Agriculture and Technology, Pantnagar, Uttarakhand, India.

\section{Insects}

S. litura larvae for isolation of gut protease and no choice feeding assay were done on an established colony of $S$. litura and $S$. obliqa, maintained at $25 \pm 1{ }^{0} \mathrm{C}, 66 \% \mathrm{RH}$ in Entomology Department, College of Agriculture G.B.P.U.A \& T Pantnagar.

\section{Chemicals}

\section{Extraction of trypsin protein}

$5 \mathrm{gm}$ leaves of soybean were grinded with the help of chilled morter and pestel and was shaken with $10 \mathrm{ml}$ of $50 \mathrm{mM}$ sodium phosphate buffer (ph 7.6) in a shaking water bath for $4 \mathrm{hr}$ at room temperature and the suspension was centrifuged at $10000 \mathrm{~g}$ for 30 min the supernatant thus obtain was used to determining the activity of trypsin inhibitor.

\section{Assay of trypsin inhibitor activity}

The activity of trypsin inhibitor was assayed by determining the residual trypsin activity following the method of Kakade et al., (1969) with slight modification using BapNA as the substrate and bovine trypsin as the standard enzyme. The reaction mixture contained $0.3 \mathrm{ml}$ diluted trypsin inhibitor (leaf extract), $0.3 \mathrm{ml} \operatorname{trypsin}(2 \mathrm{mg}$ in $40 \mathrm{ml} 0.001 \mathrm{M} \mathrm{HCL})$ and $2.1 \mathrm{ml}$ of BapNA (30 mg dissolved in minimum volume of DMSO and adjust its final volume to $100 \mathrm{ml}$ with $0.05 \mathrm{M}$ Tris $\mathrm{HCl}$, $\mathrm{pH} 8.2$, containing $0.003 \mathrm{McaCl}_{2}$ ) in a final volume of $2.7 \mathrm{ml}$.

The final concentration of BApNA in the reaction mixture was $0.54 \mathrm{mM}$ and the number at the of trypsin units was 180. After incubating the mixture at $37^{\circ} \mathrm{C}$ for $15 \mathrm{~min}$ in a shaking water bath, the reaction was stopped by adding $0.3 \mathrm{ml}$ of $30 \%$ (v/v) glacial acetic acid. A blank and a trypsin control run simultaneously. The absorbance was recorded 
at 410nm against the blank. Trypsin inhibitor activity (TIA) is defined as number of trypsin units inhibited (TUI)

\section{No choice experiment}

The antifeedant activity of 33 genotypes of soybean was evaluated against $4^{\text {th }}$ instar larvae of $S$. litura and $S$. obliqua under laboratory conditions $\left(29 \pm 5^{\circ} \mathrm{C}, \mathrm{RH} 83 \pm 5 \%\right)$ using 'no-choice' feeding technique (Belles et al., 1985 and Kumar, 1993). The fresh and matured leaves of thirty three genotypes of soybean were plucked, thoroughly washed and dried with the help of filter paper and the leaf discs $\left(\right.$ area $=4 \times 4 \mathrm{~cm}^{2}$ ) were cut from them. The leaf discs were kept in centre of pre sterilized corning glass petridishes (dia. $9 \mathrm{~cm}$ ) containing an inner lining of moist filter paper. All the treatments were replicated three times along with control. Prestarved $(3 \mathrm{~h})$ and freshly molted worms of uniform size were released in each petridish $(n=2)$ and were allowed to feed until more than $75 \%$ leaf discs were eaten away in control. The observations were recorded on leaf area consumed with the help of graph paper in the various treatments.

\section{Results and Discussion}

The activity of trypsin inhibitor was present in all the varieties but showed slight intervarietal variation and the result of Trypsin inhibitor activity (TIA) are presented in Table 1. The highest TIA activity exhibited was 2.25 TUI.mg- ${ }^{1}$ protein (SL 979) and (SL 688) followed by JS 20-41 and SL 982 with 2.23 and 2.22 TUI. mg $^{-1}$ protein respectively. The lowest TIA were recorded in CSB 904 with 0.35 TUI.mg- ${ }^{1}$ protein followed by MACS 1394 and MACS 1340 with 0.37 and 0.38 TUI.mg-1 protein respectively.

The $S$. litura gut extracts were assayed for trypsin activity by using synthetic substrates with respect to their specificities towards the protease enzyme. Specific protease activity in different cultivars fed by 4th larval stages of $S$. litura has been summarized in Table 1 . The higher gut trypsin inhibition activity ascertained in larvae fed on SL 688 with 14.40 (\%) followed by SL 979 and JS 20-41, with (14.47 and $14.22 \%)$ respectively. The lowest intensity of gut trypsin inhibition activity was observed in genotypes NRC 94 $(0.12 \%)$ followed by KDS $378(0.14)$ and KDS 378 (0.22) per cent.

In no choice feeding method for $S$. litura the minimum feeding was observed with SL 979 $\left(1.42 \mathrm{~cm}^{2}\right)$ and maximum in CSB 904 (17.48 $\mathrm{cm}^{2}$ ) over check $\left(\mathrm{Bragg}=18.77 \mathrm{~cm}^{2}\right)$, while the minimum and maximum feeding was found with SL $979\left(1.71 \mathrm{~cm}^{2}\right)$, and CSB 904 $\left(18.03 \mathrm{~cm}^{2}\right)$ respectively against larvae of $S$. obliqua over control (MLAC $\left.=18.76 \mathrm{~cm}^{2}\right)$. On the basis of preference index DS 2708, JS 2041, JS 20-69, KDS 693, KDS 705, NRC 93, RKS 113, RVS 113, RVS 2001-18, SL 979, SL 982, SL 688 and PS 1347 genotypes were found to be extremely antifeedant while DSb 19, DSb 21, MACS 1407,MACS 1416, MAUS 614, NRC 92, PS 1518, SL 958 and PS 1092 were found strongly antifeedant and DS 2706, KBS 22-2009, KDS 708 and MAUS 612 were found to be moderately antifeedant, while the remaining genotypes where found slightly antifeedant (Tables 2 and 3 ).

In the present study a fairly high degree of association was found between mean leaf area consumed and with some of important biochemical constituents in soybean genotypes (Table 4). A highly significant and negative correlation was observed between MLAC $\left(\mathrm{cm}^{2}\right)$ by $S$. litura and $S$. obliqua and protein content in 33, leaves $(\mathrm{r}=-0.728 * *)$ and $(-0.674 * *)$ respectively and trypsin inhibitor in leaves $(\mathrm{r}=-0.909 * *)$ and $(\mathrm{r}=$ $\left.0.913^{* *}\right)$. Thus it can be concluded that the 
genotypes which were having higher protein and trypsin inhibitor in their leaves offered resistance against $S$. litura and $S$. boliqua in soybean.

Table.1 Total Protein content, trypsin inhibitor activity in leaves of soybean genotypes and activity of larval gut protease of S. litura

\begin{tabular}{|c|c|c|c|c|}
\hline $\begin{array}{l}\text { Sr. } \\
\text { No. }\end{array}$ & Genotypes & $\begin{array}{c}\text { Protein } \\
(\mathrm{g} / \mathbf{1 0 0 g}) \text { Total }\end{array}$ & $\begin{array}{c}\text { Trypsine inhibitor in leaves } \\
\text { (TUI.mg }{ }^{-1} \text { protein) }\end{array}$ & $\begin{array}{l}\text { Inhibition of S.litura } \\
\text { guts protease }(\%)\end{array}$ \\
\hline 1 & CSB 904 & $21.393 \pm 0.106$ & $0.35 \pm 0.23$ & $0.21 \pm 0.29$ \\
\hline 2 & DS 2705 & $22.669 \pm 0.022$ & $0.64 \pm 0.19$ & $0.51 \pm 0.12$ \\
\hline 3 & DS 2706 & $31.750 \pm 0.017$ & $0.96 \pm 0.09$ & $2.02 \pm 0.16$ \\
\hline 4 & DS 2708 & $31.840 \pm 0.044$ & $2.19 \pm 0.11$ & $13.62 \pm 0.23$ \\
\hline 5 & DSb 19 & $33.638 \pm 0.040$ & $1.61 \pm 0.02$ & $7.03 \pm 0.09$ \\
\hline 6 & DSb 21 & $34.375 \pm 0.013$ & $1.79 \pm 0.21$ & $8.83 \pm 0.17$ \\
\hline 7 & JS-20-41 & $32.953 \pm 0.006$ & $2.23 \pm 0.13$ & $14.22 \pm 0.11$ \\
\hline 8 & JS-20-69 & $28.274 \pm 0.026$ & $1.98 \pm 0.07$ & $11.04 \pm 0.05$ \\
\hline 9 & JS-20-71 & $24.051 \pm 0.037$ & $0.71 \pm 0.03$ & $0.77 \pm 0.12$ \\
\hline 10 & KBS-22-2009 & $24.177 \pm 0.028$ & $0.92 \pm 0.14$ & $1.78 \pm 0.07$ \\
\hline 11 & KDS-378 & $24.750 \pm 0.037$ & $0.45 \pm 0.19$ & $0.14 \pm 0.13$ \\
\hline 12 & KDS-693 & $31.562 \pm 0.015$ & $2.18 \pm 0.09$ & $13.47 \pm 0.10$ \\
\hline 13 & KDS-699 & $23.449 \pm 0.071$ & $2.06 \pm 0.07$ & $12.03 \pm 0.13$ \\
\hline 14 & KDS 705 & $28.500 \pm 0.017$ & $0.79 \pm 0.12$ & $1.14 \pm 0.08$ \\
\hline 15 & KDS 708 & $23.378 \pm 0.017$ & $0.42 \pm 0.15$ & $0.22 \pm 0.19$ \\
\hline 16 & MACS 1340 & $22.598 \pm 0.034$ & $0.38 \pm 0.06$ & $0.30 \pm 0.14$ \\
\hline 17 & MACS 1394 & $22.373 \pm 0.079$ & $0.37 \pm 0.03$ & $0.32 \pm 0.18$ \\
\hline 18 & MACS 1407 & $26.677 \pm 0.002$ & $1.87 \pm 0.04$ & $9.75 \pm 0.08$ \\
\hline 19 & MACS 1416 & $27.469 \pm 0.009$ & $1.49 \pm 0.03$ & $5.93 \pm 0.11$ \\
\hline 20 & MAUS 612 & $26.151 \pm 0.170$ & $1.12 \pm 0.11$ & $3.03 \pm 0.04$ \\
\hline 21 & MAUS 614 & $27.033 \pm 0.003$ & $1.36 \pm 0.18$ & $4.82 \pm 0.07$ \\
\hline 22 & NRC 92 & $32.930 \pm 0.028$ & $1.55 \pm 0.08$ & $4.91 \pm 0.11$ \\
\hline 23 & NRC 93 & $28.156 \pm 0.030$ & $1.37 \pm 0.02$ & $6.49 \pm 0.16$ \\
\hline 24 & NRC 94 & $24.254 \pm 0.019$ & $0.50 \pm 0.05$ & $0.12 \pm 0.08$ \\
\hline 25 & PS 1518 & $23.597 \pm 0.182$ & $2.03 \pm 0.05$ & $11.64 \pm 0.17$ \\
\hline 26 & RKS 113 & $29.625 \pm 0.003$ & $1.69 \pm 0.05$ & $7.81 \pm 0.15$ \\
\hline 27 & RVS 2001-18 & $35.455 \pm 0.029$ & $2.12 \pm 0.25$ & $12.74 \pm 0.20$ \\
\hline 28 & SL 958 & $26.438 \pm 0.108$ & $2.16 \pm 0.15$ & $13.24 \pm 0.19$ \\
\hline 29 & SL 979 & $36.761 \pm 0.032$ & $2.25 \pm 0.22$ & $14.47 \pm 0.17$ \\
\hline 30 & SL 982 & $32.114 \pm 0.010$ & $2.22 \pm 0.12$ & $14.07 \pm 0.24$ \\
\hline 31 & SL 688 & $36.954 \pm 0.056$ & $2.25 \pm 0.23$ & $14.50 \pm 0.14$ \\
\hline 32 & PS 1092 & $30.060 \pm 0.046$ & $1.20 \pm 0.12$ & $3.61 \pm 0.21$ \\
\hline 33 & PS 1347 & $35.035 \pm 0.021$ & $2.20 \pm 0.18$ & $13.80 \pm 0.12$ \\
\hline
\end{tabular}


Table.2 Effect of 33 genotypes of soybean on feeding behaviour of $10 \mathrm{~d}$ old larvae of S. obliqua, Bihar hairy caterpillar

\begin{tabular}{|c|c|c|c|c|c|c|c|}
\hline $\begin{array}{l}\text { SR. } \\
\text { NO. }\end{array}$ & Genotype name & $\begin{array}{c}\text { MLAC } \\
\left(\mathbf{c m}^{2}\right)\end{array}$ & $\begin{array}{c}\text { Feeding } \\
\text { Percentage }(\%)\end{array}$ & $\begin{array}{l}\text { Antifeedant } \\
\text { Activity (\%) }\end{array}$ & $\begin{array}{c}\text { Feeding } \\
\text { Inhibition \% }\end{array}$ & $\begin{array}{l}\text { Preference } \\
\text { Index (C) }\end{array}$ & $\begin{array}{l}\text { Antifeedant } \\
\text { Category }\end{array}$ \\
\hline 1 & CSB 904 & $\begin{array}{l}18.03 \\
(4.30) \\
\end{array}$ & 72.1 & 0.79 & 2.01 & 0.98 & Slightly antifeedant \\
\hline 2 & DS 2705 & $\begin{array}{l}13.00 \\
(3.67)\end{array}$ & 51.98 & 6.155 & 18.165 & 0.82 & Slightly antifeedant \\
\hline 3 & DS 2706 & $\begin{array}{c}9.13 \\
(3.10)\end{array}$ & 36.5 & 10.28 & 34.57 & 0.65 & Moderately antifeedant \\
\hline 4 & DS 2708 & $\begin{array}{c}2.17 \\
(1.63)\end{array}$ & 8.66 & 17.705 & 79.315 & 0.21 & Extremely antifeedant \\
\hline 5 & DSb 19 & $\begin{array}{c}4.22 \\
(2.17)\end{array}$ & 16.86 & 15.515 & 63.315 & 0.36 & Strongly antifeedant \\
\hline 6 & $\mathrm{DSb} 21$ & $\begin{array}{c}4.07 \\
(2.13) \\
\end{array}$ & 16.28 & 15.67 & 64.355 & 0.35 & Strongly antifeedant \\
\hline 7 & JS 20-41 & $\begin{array}{c}1.94 \\
(1.56)\end{array}$ & 7.76 & 17.94 & 81.26 & 0.18 & Extremely antifeedant \\
\hline 8 & JS 20-69 & $\begin{array}{c}2.73 \\
(1.79)\end{array}$ & 10.92 & 17.1 & 74.62 & 0.25 & Extremely antifeedant \\
\hline 9 & JS 20-71 & $\begin{array}{l}12.71 \\
(3.63)\end{array}$ & 50.84 & 6.46 & 19.24 & 0.81 & Slightly antifeedant \\
\hline 10 & KBS 22-1009 & $\begin{array}{c}9.94 \\
(3.23)\end{array}$ & 39.76 & 9.41 & 30.745 & 0.69 & Moderately antifeedant \\
\hline 11 & KDS 378 & $\begin{array}{l}15.92 \\
(4.05) \\
\end{array}$ & 63.66 & 3.04 & 8.215 & 0.92 & Slightly antifeedant \\
\hline 12 & KDS 693 & $\begin{array}{c}2.21 \\
(1.64)\end{array}$ & 8.84 & 17.655 & 78.925 & 0.21 & Extremely antifeedant \\
\hline 13 & KDS 705 & $\begin{array}{c}2.27 \\
(1.66)\end{array}$ & 9.08 & 17.59 & 78.42 & 0.21 & Extremely antifeedant \\
\hline 14 & KDS 708 & $\begin{array}{l}10.15 \\
(3.26)\end{array}$ & 40.58 & 9.195 & 29.825 & 0.70 & Moderately antifeedant \\
\hline 15 & KDS 99 & $\begin{array}{l}15.95 \\
(4.05)\end{array}$ & 63.8 & 3 & 8.11 & 0.92 & Slightly antifeedant \\
\hline 16 & MACS 1340 & $\begin{array}{l}16.27 \\
(4.09) \\
\end{array}$ & 65.06 & 2.665 & 7.14 & 0.93 & Slightly antifeedant \\
\hline 17 & MACS 1394 & 17.00 & 67.98 & 1.89 & 4.95 & 0.95 & Slightly antifeedant \\
\hline
\end{tabular}


Int.J.Curr.Microbiol.App.Sci (2017) 6(11): 1435-1444

\begin{tabular}{|c|c|c|c|c|c|c|c|}
\hline & & $(4.18)$ & & & & & \\
\hline 18 & MACS 1407 & $\begin{array}{c}3.55 \\
(2.01) \\
\end{array}$ & 14.18 & 16.235 & 68.275 & 0.32 & Strongly antifeedant \\
\hline 19 & MACS 1416 & $\begin{array}{c}5.60 \\
(2.46)\end{array}$ & 22.4 & 14.04 & 54.035 & 0.46 & Strongly antifeedant \\
\hline 20 & MAUS 612 & $\begin{array}{c}6.77 \\
(2.69) \\
\end{array}$ & 27.08 & 12.795 & 46.98 & 0.53 & Moderately antifeedant \\
\hline 21 & MAUS 614 & $\begin{array}{c}5.78 \\
(2.50)\end{array}$ & 23.1 & 13.855 & 52.935 & 0.47 & Strongly antifeedant \\
\hline 22 & NRC 93 & $\begin{array}{c}2.32 \\
(1.68)\end{array}$ & 9.28 & 17.54 & 77.995 & 0.22 & Extremely antifeedant \\
\hline 23 & NRC 92 & $\begin{array}{c}5.47 \\
(2.44)\end{array}$ & 21.86 & 14.185 & 54.89 & 0.45 & Strongly antifeedant \\
\hline 24 & NRC 94 & $\begin{array}{l}14.86 \\
(3.91)\end{array}$ & 59.42 & 4.17 & 11.63 & 0.88 & Slightly antifeedant \\
\hline 25 & PS 1518 & $\begin{array}{c}5.89 \\
(2.52)\end{array}$ & 23.56 & 13.73 & 52.225 & 0.47 & Strongly antifeedant \\
\hline 26 & RKS 113 & $\begin{array}{c}2.25 \\
(1.65) \\
\end{array}$ & 8.98 & 17.615 & 78.63 & 0.21 & Extremely antifeedant \\
\hline 27 & RVS 2001-18 & $\begin{array}{c}1.84 \\
(1.52)\end{array}$ & 7.34 & 18.055 & 82.185 & 0.17 & Extremely antifeedant \\
\hline 28 & SL 979 & $\begin{array}{c}1.71 \\
(1.48)\end{array}$ & 6.82 & 18.195 & 83.34 & 0.16 & Extremely antifeedant \\
\hline 29 & SL 982 & $\begin{array}{c}2.11 \\
(1.61)\end{array}$ & 8.42 & 17.765 & 79.83 & 0.20 & Extremely antifeedant \\
\hline 30 & SL 958 & $\begin{array}{c}6.08 \\
(2.56)\end{array}$ & 24.3 & 13.535 & 51.095 & 0.49 & Strongly antifeedant \\
\hline 31 & SL688 & $\begin{array}{c}2.00 \\
(1.58)\end{array}$ & 7.98 & 17.885 & 80.78 & 0.19 & Extremely antifeedant \\
\hline 32 & PS1092 & $\begin{array}{c}4.17 \\
(2.16)\end{array}$ & 16.66 & 15.57 & 63.635 & 0.36 & Strongly antifeedant \\
\hline 33 & PS1347 & $\begin{array}{c}2.05 \\
(1.59)\end{array}$ & 8.18 & 17.835 & 80.345 & 0.19 & Extremely antifeedant \\
\hline 34 & BRAGG & $\begin{array}{l}18.77 \\
(4.38) \\
\end{array}$ & 75.06 & 0.00 & 0.00 & 1.00 & Preferred plant \\
\hline & CD at $5 \%$ & 0.496 & & & & & \\
\hline & F value & $* *$ & & & & & \\
\hline
\end{tabular}


Table.3 Effect of 33 genotypes on feeding behaviour of 10d old larvae of tobacco caterpillar, S. litura (Fab.)

\begin{tabular}{|c|c|c|c|c|c|c|c|}
\hline SR. NO. & Genotype name & $\begin{array}{c}\text { MLAC } \\
\left(\mathrm{cm}^{2}\right)\end{array}$ & $\begin{array}{c}\text { Feeding } \\
\text { Inhibition \% }\end{array}$ & $\begin{array}{c}\text { Feeding } \\
\text { Percentage }(\%)\end{array}$ & $\begin{array}{l}\text { Antifeedant } \\
\text { Activity (\%) }\end{array}$ & $\begin{array}{c}\text { Preference } \\
\text { Index (C) }\end{array}$ & $\begin{array}{c}\text { Antifeedant } \\
\text { Category }\end{array}$ \\
\hline 1 & CSB 904 & $\begin{array}{l}17.49 \\
(4.24) \\
\end{array}$ & 3.57 & 69.94 & 1.38 & 0.97 & Slightly antifeedant \\
\hline 2 & DS 2705 & $\begin{array}{l}12.48 \\
(3.60)\end{array}$ & 20.17 & 49.92 & 6.72 & 0.80 & Slightly antifeedant \\
\hline 3 & DS 2706 & $\begin{array}{c}7.42 \\
(2.81) \\
\end{array}$ & 43.40 & 29.68 & 12.11 & 0.57 & Moderatly antifeedant \\
\hline 4 & DS 2708 & $\begin{array}{c}1.89 \\
(1.54) \\
\end{array}$ & 81.76 & 7.54 & 18.01 & 0.18 & Extremely antifeedant \\
\hline 5 & DSb 19 & $\begin{array}{c}3.78 \\
(2.06) \\
\end{array}$ & 66.55 & 15.10 & 16.00 & 0.34 & Strongly antifeedant \\
\hline 6 & $\mathrm{DSb} 21$ & $\begin{array}{c}3.48 \\
(1.99) \\
\end{array}$ & 68.81 & 13.92 & 16.31 & 0.31 & Strongly antifeedant \\
\hline 7 & JS 20-41 & $\begin{array}{c}1.64 \\
(1.46)\end{array}$ & 83.98 & 6.54 & 18.28 & 0.16 & Extremely antifeedant \\
\hline 8 & JS 20-69 & $\begin{array}{c}2.15 \\
(1.62)\end{array}$ & 79.45 & 8.60 & 17.73 & 0.21 & Extremely antifeedant \\
\hline 9 & JS 20-71 & $\begin{array}{l}11.82 \\
(3.50) \\
\end{array}$ & 22.74 & 47.28 & 7.42 & 0.77 & Slightly antifeedant \\
\hline 10 & KBS 22-1009 & $\begin{array}{c}7.82 \\
(2.88) \\
\end{array}$ & 41.20 & 31.28 & 11.69 & 0.59 & Moderately antifeedant \\
\hline 11 & KDS 378 & $\begin{array}{l}13.81 \\
(3.78) \\
\end{array}$ & 15.27 & 55.24 & 5.29 & 0.85 & Slightly antifeedant \\
\hline 12 & KDS 693 & $\begin{array}{c}1.97 \\
(1.56)\end{array}$ & 81.05 & 7.86 & 17.93 & 0.19 & Extremely antifeedant \\
\hline 13 & KDS 705 & $\begin{array}{c}2.08 \\
(1.60) \\
\end{array}$ & 80.10 & 8.30 & 17.81 & 0.20 & Extremely antifeedant \\
\hline 14 & KDS 708 & $\begin{array}{c}8.47 \\
(2.99) \\
\end{array}$ & 37.85 & 33.88 & 10.99 & 0.62 & Moderately antifeedant \\
\hline 15 & KDS 99 & $\begin{array}{l}15.07 \\
(3.94) \\
\end{array}$ & 11.00 & 60.28 & 3.95 & 0.89 & Slightly antifeedant \\
\hline 16 & MACS 1340 & $\begin{array}{l}15.19 \\
(3.96) \\
\end{array}$ & 10.56 & 60.76 & 3.83 & 0.90 & Slightly antifeedant \\
\hline 17 & MACS 1394 & $\begin{array}{l}15.55 \\
(4.00)\end{array}$ & 9.42 & 62.20 & 3.44 & 0.91 & Slightly antifeedant \\
\hline 18 & MACS 1407 & $\begin{array}{c}2.51 \\
(1.73)\end{array}$ & 76.46 & 10.02 & 17.35 & 0.24 & Extremely antifeedant \\
\hline 19 & MACS 1416 & $\begin{array}{c}4.65 \\
(2.26) \\
\end{array}$ & 60.36 & 18.58 & 15.07 & 0.40 & Strongly antifeedant \\
\hline 20 & MAUS 612 & 5.95 & 51.87 & 23.80 & 13.68 & 0.48 & Strongly antifeedant \\
\hline
\end{tabular}


Int.J.Curr.Microbiol.App.Sci (2017) 6(11): 1435-1444

\begin{tabular}{|c|c|c|c|c|c|c|c|}
\hline & & $\begin{array}{l}(2.53) \\
\end{array}$ & & & & & \\
\hline 21 & MAUS 614 & $\begin{array}{c}5.03 \\
(2.35)\end{array}$ & 57.74 & 20.12 & 14.66 & 0.43 & Strongly antifeedant \\
\hline 22 & NRC 93 & $\begin{array}{c}2.13 \\
(1.62)\end{array}$ & 79.67 & 8.50 & 17.76 & 0.21 & Extremely antifeedant \\
\hline 23 & NRC 92 & $\begin{array}{c}4.32 \\
(2.19)\end{array}$ & 62.69 & 17.26 & 15.42 & 0.37 & Strongly antifeedant \\
\hline 24 & NRC 94 & $\begin{array}{l}13.05 \\
(3.68)\end{array}$ & 18.03 & 52.20 & 6.11 & 0.82 & Slightly antifeedant \\
\hline 25 & PS 1518 & $\begin{array}{c}5.18 \\
(2.38)\end{array}$ & 56.76 & 20.72 & 14.50 & 0.31 & Strongly antifeedant \\
\hline 26 & RKS 113 & $\begin{array}{c}2.04 \\
(1.59)\end{array}$ & 80.45 & 8.14 & 17.85 & 0.20 & Extremely antifeedant \\
\hline 27 & RVS 2001-18 & $\begin{array}{c}1.54 \\
(1.42)\end{array}$ & 84.84 & 6.16 & 18.38 & 0.15 & Extremely antifeedant \\
\hline 28 & SL 979 & $\begin{array}{c}1.42 \\
(1.38) \\
\end{array}$ & 85.98 & 5.66 & 18.52 & 0.14 & Extremely antifeedant \\
\hline 29 & SL 982 & $\begin{array}{c}1.84 \\
(1.52)\end{array}$ & 82.20 & 7.34 & 18.07 & 0.18 & Extremely antifeedant \\
\hline 30 & SL 958 & $\begin{array}{c}5.72 \\
(2.49)\end{array}$ & 53.30 & 22.88 & 13.92 & 0.47 & Strongly antifeedant \\
\hline 31 & SL688 & $\begin{array}{c}1.75 \\
(1.49)\end{array}$ & 83.00 & 6.98 & 18.16 & 0.17 & Extremely antifeedant \\
\hline 32 & PS1092 & $\begin{array}{c}3.51 \\
(2.00)\end{array}$ & 68.58 & 14.04 & 16.28 & 0.39 & Strongly antifeedant \\
\hline 33 & PS1347 & $\begin{array}{c}1.78 \\
(1.50)\end{array}$ & 82.73 & 7.10 & 18.13 & 0.23 & Extremely antifeedant \\
\hline 34 & BRAGG & $\begin{array}{l}18.78 \\
(4.38)\end{array}$ & 0.00 & 75.10 & 0.00 & 1.00 & Preferred plant \\
\hline & CD at $5 \%$ & 0.554 & & & & & \\
\hline & $F$ value & $* *$ & & & & & \\
\hline
\end{tabular}

Table.4 Simple correlation coefficient between biochemical constituents of soybean genotypes leaves and mean leaf area consumed

\begin{tabular}{|c|c|c|}
\hline Chemical compounds & $\begin{array}{c}\text { Mean leaf area consumed by } \boldsymbol{S} . \\
\text { litura }\end{array}$ & Mean leaf area consumed by S. obliqua \\
\hline Proteins & $-0.728^{* *}$ & $-0.674^{* *}$ \\
\hline Trypsin inhibitor & $-0.909^{* *}$ & $-0.913^{* *}$ \\
\hline
\end{tabular}

** Highly significant at $1 \%$ 
Other authors also found that protease inhibitors play important role in plant defense mechanism by preventing proteolysis in the midgut of insect larvae leading to their starvation and subsequent death (Gatehouse et al., 1999). This fact can be interpreted as a potential strategy for increasing the level of plant defense against insects (Kansal et al., 2008).

Manus and Burgess (1995) reported that the extracts from the digestive tract of final instar larvae of Spoduptera litura, reared on artificial diet, contained trypsin activity with a $\mathrm{pH}$ optimum of 10.5, an elastase activity with a $\mathrm{pH}$ optimum of 10.5 and leucine aminopeptidase activity with a $\mathrm{pH}$ optimum of 8.5. No chymotrypsin amidase activity could be detected, but chymotrypsin esterase activity was measured with a $\mathrm{pH}$ optimum of 8.5. Titration, in uitvo of the soybean (Kunitz) trypsin inhibitor (SBTI) against each enzyme at its optimal $\mathrm{pH}$ revealed that the inhibitor was most effective at retarding the trypsinlike activity, only slightly effective against the elastase-like and chymotrypsin esterase-like activity, but completely ineffective against leucine aminopeptidase. Incorporation of SBTI into the diet of neonate larvae at $0.2 \%$ $(\mathrm{w} / \mathrm{v})$ and $0.5 \%(\mathrm{w} / \mathrm{v})$ retarded growth rate when compared with larvae fed artificial diet only. Franco et al., (2004) reported that Enzymatic assays using gut extracts from larval and adult boll weevil have demonstrated the presence of digestive serine proteinase-like activities and in vitro assays showed that soybean Kunitz trypsin inhibitor (SKTI) was able to inhibit these enzymes.

Gholamzadeh et al., (2013) investigated that, the Proteolytic activity of azocasein as a protein substrate in the gut of $C$. nemorana was $7.267 \pm 0.37 \mu \mathrm{mol} / \mathrm{min} / \mathrm{mg}$ protein. In addition, the trypsin and chymotrypsin activity was $1.53 \pm 0.03$ and $1.42 \pm 0.1$ $\mu \mathrm{mol} / \mathrm{min} / \mathrm{mg}$ protein, respectively. The azocasein hydrolysis by the midgut extract of A. janata resulted in the specific activity of $1200 \pm 90 \mathrm{nmol} / \mathrm{min} / \mathrm{mg}$ protein (Budatha et al., 2008).

A highly significant negative correlation was observed between mean leaf area consumed $\left(\mathrm{cm}^{2}\right)$ by $S$. litura and $S$. obliqua and protein content in 33 genotypes leaves $(\mathrm{r}=-0.728 * *)$ and $(-0.674 * *)$ respectively and trypsin inhibitor in leaves $\left(\mathrm{r}=-0.909^{* *}\right)$ and $(\mathrm{r}=$ $\left.0.913^{* *}\right)$. Thus it can be concluded that the genotypes which were having higher protein and trypsin inhibitor in their leaves offered resistance against $S$. litura and $S$. boliqua in soybean crop.

\section{Acknowledgements}

Authors are grateful to "Indian Council of Medical Research (ICMR)" for awarding Senior Research fellowship to first author for her Ph.D. research. Authors are also thankful to Dean Agriculture, Govind Ballabh Pant University of Agriculture and Technology, Pantnagar, for providing the necessary facilities and support for carrying out the study successfully.

\section{References}

Ahmad, Z., Saleemuddin, M., and Siddi, M. (1980). Purification and characterisation of three alkaline proteases from the gut of the larva of army worm, Spodoptera litura. Insect Biochemistry, 10: 667-673.

Belles, X., Camps, F., Coil, J. and Piulachs, M. D. 1985. Insect antifeedant activity of clerodane diterpenoids against larvae of Spodoptera littoralis (Boisd.) (Lepidoptera). Journal of Chemical Ecology, 11:1439-1445.

Birk, Y. 1995. Protein proteinase inhibitors in legume seeds: Overview. Arch. Latinoam. Nutr. 44: 26-30.

Budatha M., Meur, G. and Dutta-Gupta, A., 2008. Identification and characterization of midgut proteases in Achaea janata and their 
implications. Biotechnology Letters. 30: 305-310.

Franco, O.V., Dias, C. S., Magalha, P.C., Monteiro, A.C.S., Bloch, C., Francislete, R.M. and Osmundo, B. O. 2004. Effects of soybean Kunitz trypsin inhibitor on the cotton boll weevil (Anthonomus grandis). Phytochemistry 65 81-89.

Gangrade, G.A. 1976. Assessment of effect on yield and quality of soybean caused by major arthropod pest, Terminals Technical Report on the project, J.N.K.V.V., Jabalpur, M.P. India. 143p.

Gatehouse, A.M.R., Norton E., Davison G.M., Babbe S.M., Newell C.A. and Gatehouse J.A. 1999. Digestive proteolytic activity in larvae of tomato moth, Lacanobia oleracea, effects of plant proteinase inhibitor in vitro and in vivo. J. Insect Physiol., 45: 545-558.

Gholamzadeh, C.M., Ghadamyari, M. and Sharifi, M. 2013. Identification and Characterisation of Gut Proteases in the Fig Tree Skeletoniser Moth, Choreutis nemorana Hübner (Lepidoptera: Choreutidae). Plant Protect. Sci., 49(1): 19-26.

Hamad, M.B.B., and Attias J. (1987). Isolation and partial characterization of two alkaline proteases of the greater wax moth Galleria mellonella L. Insect Biochemistry, 17: 653658.

Houseman J.G., Downe A.E.R., and Philogene B.J.R. (1989). Partial characterization of proteinase activity in the larval midgut of the European corn borer, Ostrinia nubilalis Hubner (Lepidoptera: Pyrolidae). Canadian Journal of Zoology, 67: 864-868.

Johnstone, C. and Thompson, 1991. A simple and rapid method for preparation of plant genome DNA for PCR analysis. Nucl. Acid Res., 19:18-29.
Kakade. M.L., Simons, N. and Llener, I.E. 1969. An evaluation of natural vs synthetic substrates for measuring the antitryptic activity of soybean samples. Cereal Chem., 46: 518.

Kansal, R., Gupta, R.N., Koundal, K.R., Kuhar, K. and Gupta, V.K. 2008. Purification, characterization and evaluation of insecticidal potential of trypsin inhibitor from mungbean (Vigna radiata $\mathrm{L}$. Wilczek) seeds. Acta Physiol. Plant., 30: 761-768.

Koiwa, H., Bressan, A. and Hasegawa, P.M. 1997. Regulation of protease inhibitors and plant defense. Trends in Plant Science. 2: 379384.

Kumar, S. 1993. Feeding deterrent and insecticidal activity of weed plants from tarai region against Henosepilachna vignitioctopuntata (Fab.). Thesis, M.Sc. (Ag.), G.B. Pant University of Agriculture and Technology, Pantnagar, pp 82.

Manus, T.M. and Burgess, E.P.J. 1995. Effects of the soybean (Kunitz) trypsin inhibitor on growth and digestive proteases of larvae of Spodoptera litura. J. Insect Physiology. 41(9): 731-738.

Sasaki, T., and Suzuki, Y. (1982). Alkaline proteases in digestive juice of the silk worm, Bombyx mori. Biochimica et Biophysica Acta, 703: 1-10.

Schaller, A. and Ryan, C.A. 1995. Systemin- a polypeptide defense signal in plants. Bioessays., 18: 27-33.

Shukle, R.H., and Murdock, L.L. (1983). Lipoxygenase, trypsin inhibitor, and lectin from Soybeans: Effects on larval growth of Manduca sexta (Lepidoptera: Sphingidae). Environmental Entomology, 12: 787-791.

\section{How to cite this article:}

Anchala Nautiyal, Neeta Gaur and Kamendra Singh. 2017. Effect of Soybean Leaf Protease Inhibitor on the Mean Leaf Area Consumed by Spodopetra litura and Spilosoma obliqua larvae. Int.J.Curr.Microbiol.App.Sci. 6(11): 1435-1444.

doi: https://doi.org/10.20546/ijcmas.2017.611.171 